\title{
Coronary artery bypass graft surgery in a pediatric patient with a giant coronary aneurysm
}

\author{
Mete Gürsoy, ${ }^{1}$ Ece Salihoğglu, ${ }^{2}$ Salih Özçobanoğlu, ${ }^{2}$ Süleyman Özkan ${ }^{2}$ \\ Received: February 07, 2014 Accepted: August 21, 2014 Published online: February 10, 2015
}

Kawasaki disease or mucocutaneous lymph node syndrome is an acute, febrile vasculitis of unknown etiology affecting mainly infants and children under five years of age. Coronary artery involvement, which occurs in approximately $20 \%$ of such patients, may be the most important feature of this syndrome. In this paper, we report a successful coronary artery bypass graft in a nine-year-old boy with a giant coronary artery aneurysm and diffuse coronary artery disease.

Keywords: Aneurysm; coronary artery bypass grafting; Kawasaki disease.

Kawasaki disease (KD), previously known as mucocutaneous lymph node syndrome, is an acute, febrile, self-limiting generalized vasculitis of unknown etiology which occurs predominantly in infants and young children. It mainly affects small- and medium-sized arteries, particularly the coronary arteries, and was described by Kawasaki in 1967..$^{[1]}$ Gulhan et al. ${ }^{[2]}$ reported a $42.4 \%$ incidence of coronary involvement in Turkish patients with KD. Kawasaki disease causes aneurysm formation at the proximal sites of the coronaries, while the distal sites are usually protected. Dilatation equal to or larger than $8 \mathrm{~mm}$ is categorized as a giant aneurysm, which may cause coronary artery stenosis secondary to compression or thrombosis. ${ }^{[3]}$ Early diagnosis and rapid therapeutic interventions such as aspirin and intravenous gamma globulin may decrease the risk of coronary artery pathology development by approximately $20 \%$. $^{[4]}$

In this paper, we present a successful coronary artery bypass grafting (CABG) in a boy who had one of the largest reported childhood coronary artery aneurysms to date.

\section{CASE REPORT}

A nine-year-old boy with KD was admitted to our institution with complaints of chest pain and fatigue. The patient's history revealed KD. He experienced the first attack at the age of two with prolonged fever. Neither intravenous gamma globulin nor steroid therapy was given. Laboratory examination showed leukocytosis, high C-reactive protein, and elevated liver enzymes. Echocardiography showed pancarditis and a giant calcified and thrombotic coronary artery aneurysm. The diameter of the left main coronary artery aneurysm was measured as $40 \mathrm{~mm}$. The patient was enrolled in a treadmill exercise test according to the modified Bruce protocol. Ischemic signs were found in the anterior leads. Coronary angiography revealed a giant left main coronary artery aneurysm, a totally occluded left anterior descending artery, and a diffusely diseased circumflex artery (Figure 1). The patient underwent $\mathrm{CABG}$ with upper mini-sternotomy. Perioperatively, the giant calcified coronary aneurysm was seen between the aorta and the pulmonary artery (Figure 2). Myocardial protection was achieved with antegrade cold blood cardioplegia and topical cooling. In situ, the left internal thoracic artery -the first obtuse margin branch of the circumflex artery, and the right internal thoracic artery- the left anterior descending artery anastomoses were performed under cardiopulmonary bypass. The aneurysm wall was calcified and non-resectable. The postoperative course was uneventful. Dual antiplatelet therapy was started and the patient was discharged at the postoperative seventh day in a good condition. The patient's

${ }^{1}$ Department of Cardiovascular Surgery, Acıbadem University Bahçelievler Family Hospital, İstanbul, Turkey

${ }^{2}$ Department of Pediatric Cardiac Surgery, Acıbadem University International Hospital, İstanbul, Turkey

Corresponding author: Mete Gürsoy, M.D. Denizatı Sitesi, A-4 Blok, No: 23, 34025 Zeytinburnu, İstanbul, Turkey.

Tel: +90 505 - 6791484 e-mail: metegursoy35@gmail.com 


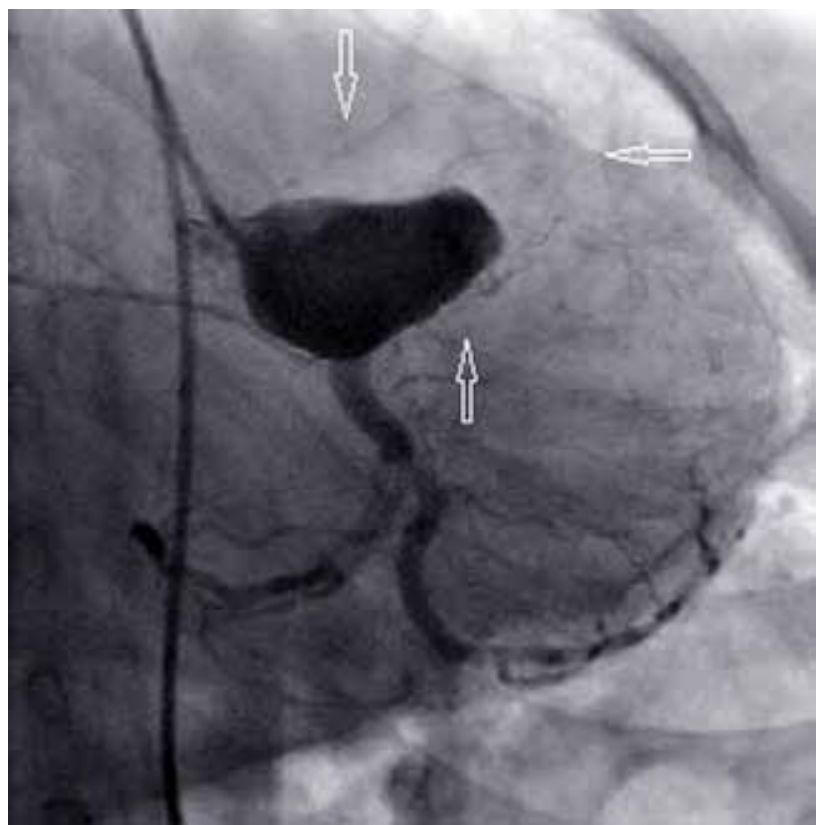

Figure 1. Coronary angiography showing partially thrombosed aneurysm, left anterior descending artery and circumflex artery with involvement (arrows show calcific wall of the aneurysm).

cardiologic follow-up data was reviewed retrospectively and the postoperative course was found uneventful without angina pectoris and ideal functional capacity.

\section{DISCUSSION}

Coronary artery aneurysm occurs in approximately $20 \%$ of untreated patients with $\mathrm{KD}$, whereas it accounts for only $4 \%$ to $8 \%$ of children who receive intravenous gamma globulin. ${ }^{[4]}$ Currently, early high-dose intravenous gamma globulin is accepted as the standard initial therapy. Late intravenous gamma globulin administration may also decrease inflammation, but may not be able to prevent coronary artery lesions. ${ }^{[5,6]}$ In the presence of clinical suspicion, persisting fever less than five days may be considered to be a positive diagnostic criterion and intravenous gamma globulin can be employed. ${ }^{[7]}$ Chen et al. ${ }^{[8]}$ analyzed nine studies investigating the progression of coronary involvement in $\mathrm{KD}$ and concluded that the combination of a corticosteroid with the conventional regimen of intravenous gamma globulin as the initial treatment strategy might reduce the risk of coronary abnormality. ${ }^{[8,9]}$ Currently, TNF alpha antagonists are used, particularly in patients with intravenous gamma globulin resistance. ${ }^{[10]}$ Therefore, our patient was

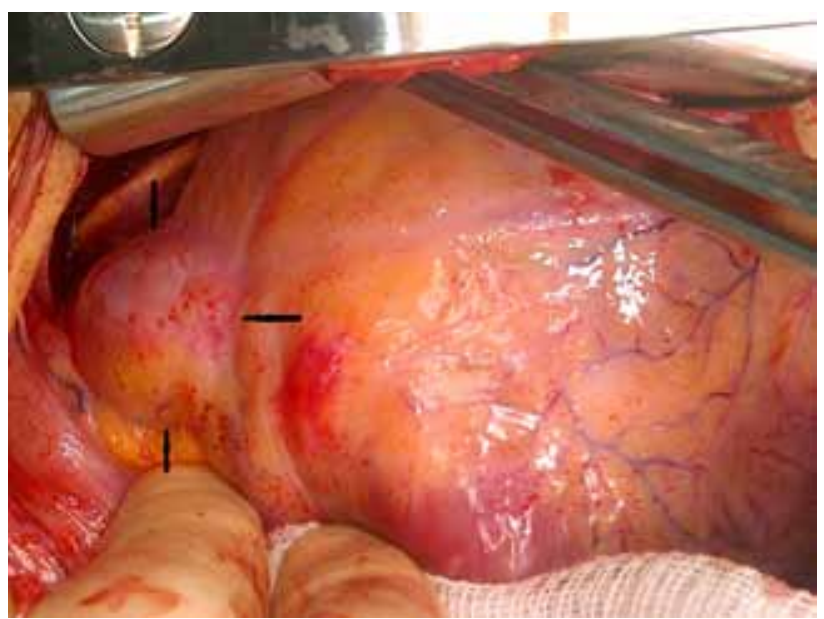

Figure 2. Perioperative appearance of proximal coronary artery aneurysm (arrows show aneurysm).

considered inappropriate for TNF alpha antagonist therapy due to late presentation.

Kawasaki disease generally affects proximal segments of the coronaries. In our patient, the left anterior descending artery was diffusely diseased and the circumflex artery was segmentally involved. Our patient received neither immunoglobulin nor steroid therapy previously and experienced prolonged periods of fever several times. The association of prolonged fever with coronary artery lesions has been well established. ${ }^{[11]}$ Frequent cardiac imaging in fever episodes may be helpful, since children cannot distinguish ischemic symptoms.

Although coronary aneurysms due to KD may be asymptomatic, myocardial infarction and rupture are life-threatening complications which are rarely reported in the literature. Aneurysms may cause thrombosis, distal embolization, calcification, coronary stenosis, myocardial ischemia, infarction, rupture, cardiac tamponade and even death. Akagi et al. ${ }^{[12]}$ reported a $1.5 \%$ incidence of myocardial infarction in patients with a giant coronary artery aneurysm during a four-year follow-up period. Coronary aneurysms do not tend to rupture except in acute illness. Ruptured aneurysms have been noted as case reports and, to the best of our knowledge, there is no satisfactory study investigating the incidence and risk factors for aneurysm rupture. The selection of the ideal surgical technique is still controversial. In symptomatic patients, CABG with or without aneurysmectomy is the preferred approach. Aneurysmectomy can be 
performed in selected cases, however, it may cause difficulties in revascularization and the protection of branches. On the other hand, ligation of the aneurysm and distal bypass is a controversial alternative due to the risk of subsequent graft failure. ${ }^{[13]}$ Coronary artery bypass graft surgery with internal thoracic artery graft is the gold standard in patients suffering from myocardial ischemia. ${ }^{[13]}$ The internal thoracic artery is the preferred graft with a high long-term patency rate. ${ }^{[13]}$ In our patient, we utilized bilateral internal thoracic artery grafts for revascularization.

In conclusion, giant coronary aneurysm is a very rare and life-threatening complication of KD. Early diagnosis and aggressive treatment may prevent aneurysm formation. Coronary artery bypass grafting is a safe and preferred approach with encouraging long-term results in patients suffering from myocardial ischemia.

\section{Declaration of conflicting interests}

The authors declared no conflicts of interest with respect to the authorship and/or publication of this article.

\section{Funding}

The authors received no financial support for the research and/or authorship of this article.

\section{REFERENCES}

1. Kawasaki T. Acute febrile mucocutaneous syndrome with lymphoid involvement with specific desquamation of the fingers and toes in children. Arerugi 1967;16:178-222.

2. Gülhan B, Kesici S, Beken S, Cilsal E, Kale G, Alehan D, et al. Varying clinical features of Turkish Kawasaki disease patients. Turk J Pediatr 2012;54:1-6.
3. Onouchi Z, Hamaoka K, Sakata K, Ozawa S, Shiraishi I, Itoi $\mathrm{T}$, et al. Long-term changes in coronary artery aneurysms in patients with Kawasaki disease: comparison of therapeutic regimens. Circ J 2005;69:265-72.

4. Yanagawa H, Yashiro M, Nakamura Y, Kawasaki T, Kato H. Results of 12 nationwide epidemiological incidence surveys of Kawasaki disease in Japan. Arch Pediatr Adolesc Med 1995;149:779-83.

5. Weng KP, Ou SF, Lin CC, Hsieh KS. Recent advances in the treatment of Kawasaki disease. J Chin Med Assoc 2011;74:481-4.

6. Muta $H$, Ishii $M$, Yashiro $M$, Uehara R, Nakamura $Y$. Late intravenous immunoglobulin treatment in patients with Kawasaki disease. Pediatrics 2012;129:e291-7.

7. Ayusawa M, Sonobe T, Uemura S, Ogawa S, Nakamura $\mathrm{Y}$, Kiyosawa N, et al. Revision of diagnostic guidelines for Kawasaki disease (the 5th revised edition). Pediatr Int 2005;47:232-4.

8. Chen S, Dong Y, Yin Y, Krucoff MW. Intravenous immunoglobulin plus corticosteroid to prevent coronary artery abnormalities in Kawasaki disease: a meta-analysis. Heart 2013;99:76-82.

9. Wooditch AC, Aronoff SC. Effect of initial corticosteroid therapy on coronary artery aneurysm formation in Kawasaki disease: a meta-analysis of 862 children. Pediatrics 2005;116:989-95.

10. Son MB, Gauvreau K, Burns JC, Corinaldesi E, Tremoulet $\mathrm{AH}$, Watson $\mathrm{VE}$, et al. Infliximab for intravenous immunoglobulin resistance in Kawasaki disease: a retrospective study. J Pediatr 2011;158:644-9.

11. Ram Krishna M, Sundaram B, Dhanalakshmi K. Predictors of coronary artery aneurysms in Kawasaki disease. Clin Pediatr (Phila) 2014;53:561-5.

12. Akagi T, Rose V, Benson LN, Newman A, Freedom RM. Outcome of coronary artery aneurysms after Kawasaki disease. J Pediatr 1992;121:689-94.

13. Kitamura S, Tsuda E, Kobayashi J, Nakajima H, Yoshikawa Y, Yagihara T, et al. Twenty-five-year outcome of pediatric coronary artery bypass surgery for Kawasaki disease. Circulation 2009;120:60-8. 